Case Report

\title{
Antiproteinase 3 Positive Eosinophilic Granulomatosis with Polyangiitis Presenting with Heart Failure and Intraventricular Thrombosis
}

\author{
Dan Zhu, ${ }^{1}$ Yiming Luo, ${ }^{2}$ Xiangyuan Liu, ${ }^{3}$ and Lingyun $\mathrm{Zu}^{1}$ \\ ${ }^{1}$ Department of Cardiology, Peking University Third Hospital, Key Laboratory of Cardiovascular Molecular Biology and \\ Regulatory Peptides, Ministry of Health, Beijing, China \\ ${ }^{2}$ Department of Medicine, Mount Sinai St. Luke's and Mount Sinai West Hospitals, Icahn School of Medicine at Mount Sinai, \\ New York, NY, USA \\ ${ }^{3}$ Department of Rheumatology and Immunology, Peking University Third Hospital, Beijing, China
}

Correspondence should be addressed to Lingyun Zu; dr_zly@126.com

Received 22 August 2016; Revised 7 November 2016; Accepted 5 December 2016; Published 29 January 2017

Academic Editor: Jamal Mikdashi

Copyright (C) 2017 Dan Zhu et al. This is an open access article distributed under the Creative Commons Attribution License, which permits unrestricted use, distribution, and reproduction in any medium, provided the original work is properly cited.

\begin{abstract}
Eosinophilic granulomatosis with polyangiitis (EGPA) is a rare systemic vasculitis commonly with cardiac complications. We describe a case of anti-PR3 ANCA-positive EGPA complicated by congestive heart failure and intraventricular thrombosis. Interestingly, the thrombus was resolved rapidly with steroid and cyclophosphamide in the setting of interrupted anticoagulation. To the best of our knowledge, we report the first case of anti-PR3 positive EGPA with extensive cardiac involvement. Our patient had overlapping features with previously studied ANCA-positive and ANCA-negative EGPA cases. We also hypothesize that the thrombogenic potential of eosinophils may play a central role in thrombogenesis in EGPA and aggressive immunosuppressive therapy remains the cornerstone of treatment, and the addition of anticoagulation therapy in the setting of thrombus formation and also very high risk of bleeding needs to be considered cautiously.
\end{abstract}

\section{Introduction}

Eosinophilic granulomatosis with polyangiitis (EGPA), formerly named Churg-Strauss syndrome, is a rare systemic vasculitis of small- and medium-sized vessels, with heart involvement being a common complication. We present a case of a 20-year-old male with antiproteinase 3 (PR3) antineu trophil cytoplasmic antibodies (ANCA) positive EGPA complicated by severe cardiomyopathy and intraventricular thrombosis, which responded robustly to aggressive immunosuppressive therapy with successful thrombolysis in the setting of interrupted anticoagulation therapy.

\section{Case Presentation}

A 20-year-old male with a 6-month history of chronic cough, progressive exertional dyspnea, and recurrent epistaxis was admitted for worsening dyspnea. He also complained of right lower extremity burning pain and numbness. On physical examination, he had generalized lymphadenopathy, S3 gallop, bilateral pulmonary crackles, hepatomegaly, and peripheral edema. No skin rash was noted. Neurological examination revealed flattening of the nasolabial fold, decreased sensation over the right lower extremity, and generalized hypoactive tendon reflex. Complete blood count showed elevated eosinophil count of $7390 \mathrm{n} / \mu \mathrm{L}$, which contributed to $28.1 \%$ of leukocytes. Erythrocyte sedimentation rate was $69 \mathrm{~mm} / \mathrm{h}$. N-terminal of the prohormone brain natriuretic peptide (NT-proBNP) was $27523 \mathrm{pg} / \mathrm{mL}$ upon admission. Liver function tests showed alanine transaminase $1441 \mathrm{u} / \mathrm{L}$, total bilirubin $29.0 \mu \mathrm{mol} / \mathrm{L}$, and albumin $23.9 \mathrm{~g} / \mathrm{L}$. Urinalysis and creatinine were normal. Immunological workup showed positive ANCA with anti-PR3 level of $91 \mathrm{IU} / \mathrm{mL}$ but negative for antimyeloperoxidase (MPO), as well as an IgE level of above $2500 \mathrm{IU} / \mathrm{mL}$. Antinuclear antibodies and rheumatoid factor were negative. Electrocardiogram demonstrated nonspecific ST-T wave changes. Serial chest X-ray in the hospital 


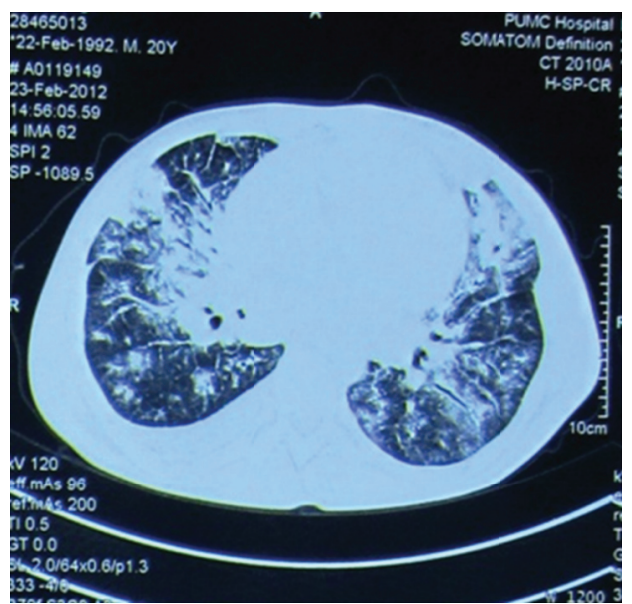

FIGURE 1: Chest CT. It shows bilateral patchy ground-glass opacities and subpleural nodules.

showed migratory infiltrative changes compared to the one from outside the hospital three weeks before. Chest CT scan showed bilateral ground-glass opacities and multiple subpleural nodules (Figure 1). Transthoracic Echocardiogram (TTE) demonstrated four-chamber dilatation, decreased wall motion, and apical intramural thrombus, with left ventricular ejection fraction (LVEF) of 26\% (Figure 2). Nasal polyps were detected by rhinoscopy and excisional biopsy showed small amount of intraepithelial eosinophilic infiltration and subepithelial amyloid deposition. Bone marrow biopsy was negative for leukemia and also with negative FIP1L1-PDGFRA and PDGFRB fusion genes. Based on the above clinical, laboratory, and imaging findings, a diagnosis of EGPA was made.

The patient was treated with pulse methylprednisolone $500 \mathrm{mg}$ intravenously daily for 5 days and then gradually tapered to $60 \mathrm{mg}$ orally daily and cyclophosphamide $400 \mathrm{mg}$ intravenously once, followed by $100 \mathrm{mg}$ orally daily. Warfarin was also started with enoxaparin bridging therapy. His heart failure was managed by aggressive diuresis. His hospital course was complicated by upper gastrointestinal tract bleeding at day 4 so anticoagulants were discontinued. Repeated TTE at day 7 revealed complete resolution of intraventricular thrombus; at the same time, his eosinophil count was lowered to 0 . He was discharged after significant symptomatic improvement and his LV EF was back to $34 \%$.

\section{Discussion}

We described a case of a young male who presented with severe cardiomyopathy with intraventricular thrombus, clinical peripheral neuropathy, nasal polyps, pulmonary nodules, hepatitis, eosinophilia, and anti-PR3 ANCA positive. The diagnostic discrimination between EGPA and hypereosinophilic syndrome (HSE) can be challenging, especially in the absence of histologically proven vasculitis. The absence of FIPIL1-PDGFRA and PDGFRB gene fusion and unremarkable bone marrow biopsy ruled out most clonal forms of

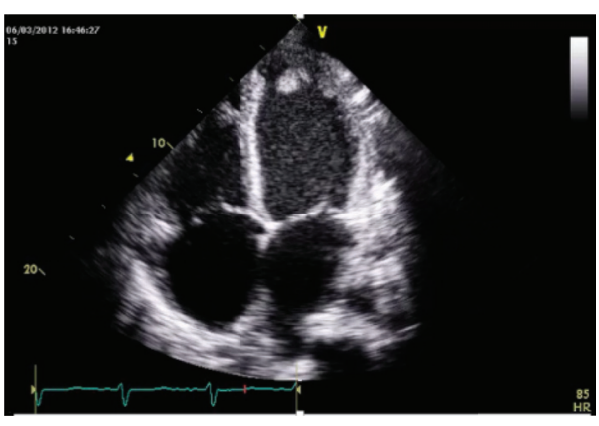

FIgURE 2: Transthoracic Echocardiogram (TTE). A left ventricular apical intramural thrombus can be visualized.

HSE. Positive ANCA can be used as a surrogate marker for vasculitis [1]. And our case fits into the American College of Rheumatology 1990 classification criteria [2]. Therefore, we consider our case a diagnosis EGPA. In clinical practice, a histological diagnosis may not always be possible and the classic pathological triad of necrotizing vasculitis, eosinophilic infiltration, and extravascular granuloma rarely coexisted in any one patient [3]. At the meantime, anti-PR3 ANCA is very rare in EGPA [4] and false positive ANCA, although also rare, is possible [5]. Thus, our case calls for a both accurate and practical classification and diagnostic criteria to aid clinical judgement and better understand the utility of surrogate markers in the setting of atypical presentations. Currently, a multinational observational study is underway for this challenge [6].

Due to the heterogeneity of EGPA, efforts have been made to identify its subtypes. Unlike granulomatosis with polyangiitis (GPA) and microscopic polyangiitis (MPA), only $40 \%$ of EGPA is ANCA-positive. Studies $[4,7]$ have suggested a phenotypic distinction between ANCA-positive and ANCA-negative EGPA, with the former associated with renal involvement, peripheral neuropathy, and biopsy-proven vasculitis and the latter associated with higher amount of eosinophilia and cardiac involvement. However, those studies mainly included patients with anti-MPO ANCA. To the best of our knowledge, we report the first case of anti-PR3 ANCA-positive EGPA with extensive cardiac involvement. And our patient had overlapping features among previously studied ANCA-positive and ANCA-negative EGPA cases [4, 7]. Due to its rarity, how to incorporate anti-PR3 ANCA positivity into subcategorization of EGPA warrants further investigations.

Cardiac involvement is well documented in patients with EGPA, with incidence ranging from $17 \%$ to $92 \%$ depending on the diagnostic modality and accounting for almost 50\% mortality in EGPA. Cardiac involvement is also included in the Five-Factor Score (FFS) which predicts a worse outcome and an indication for adjunct cytotoxic treatment with cyclophosphamide in addition to systemic glucocorticoid [8]. Although this recommendation was based on expert opinion [3], Miszalski-Jamka et al. [9] found that lack of noncorticosteroid immunosuppression is an independent determinant of cardiac involvement in EGPA and the extent 
of myocardial damage is associated with insufficient duration of noncorticosteroid immunosuppression.

Intraventricular thrombosis has been reported in patients with EGPA and was presumably caused by eosinophilic endocarditis [10]. The underlying pathophysiology of cardiac thrombus in patients with EGPA is unknown. It has been shown that eosinophils may play an important role in thrombosis especially in hypereosinophilic disorders, involving enhanced tissue factor expression [11] and generating prothrombotic fibrin structure [12]. We demonstrated complete resolution of intraventricular thrombus within one week following aggressive immunosuppressive therapy in the setting of interrupted anticoagulation therapy. It is possible that treating eosinophilia removed the driving factor of thrombogenesis and subsequently led to rapid thrombolysis. Complete resolution of intraventricular thrombus by aggressive immunosuppression without anticoagulation in systemic vasculitis has been reported in literature [13, 14], including one case of EGPA [14]. Although there is no consensus over this particular setting, the selection and duration of anticoagulation therapy need to be considered more cautiously in patients with very high risk of bleeding or the occurrence of clinical bleeding is uncertain but cannot be ruled out.

In conclusion, we present a case of anti-PR3 ANCApositive EPGA with extensive cardiac involvement, which responded rapidly with aggressive immunosuppressive therapy. The atypical clinical presentation calls for more accurate and practical classification and diagnostic criteria. The biological and clinical significance of anti-PR 3 ANCA in EGPA is not clear. And the optimal duration of anticoagulation therapy for thrombosis in systemic vasculitis treated with aggressive immunosuppressive therapy warrants more investigations.

\section{Competing Interests}

The authors declare that there is no conflict of interests regarding the publication of this paper.

\section{Authors' Contributions}

Dan Zhu and Yiming Luo contributed equally to this article.

\section{Acknowledgments}

This work is supported by grants from the National Natural Science Foundation of China (81200167) and the Ph.D. Programs Foundation of Ministry of Education of China (20120001120131).

\section{References}

[1] R. Watts, S. Lane, T. Hanslik et al., "Development and validation of a consensus methodology for the classification of the ANCAassociated vasculitides and polyarteritis nodosa for epidemiological studies," Annals of the Rheumatic Diseases, vol. 66, no. 2, pp. 222-227, 2007.
[2] A. T. Masi, G. G. Hunder, J. T. Lie et al., "The American college of rheumatology 1990 criteria for the classification of churg-strauss syndrome (allergic granulomatosis and angiitis)," Arthritis and Rheumatism, vol. 33, no. 8, pp. 1094-1100, 1990.

[3] A. Mahr, F. Moosig, T. Neumann et al., "Eosinophilic granulomatosis with polyangiitis (Churg-Strauss): evolutions in classification, etiopathogenesis, assessment and management," Current Opinion in Rheumatology, vol. 26, no. 1, pp. 16-23, 2014.

[4] C. Comarmond, C. Pagnoux, M. Khellaf et al., "Eosinophilic granulomatosis with polyangiitis (Churg-Strauss): clinical characteristics and long-term followup of the 383 patients enrolled in the French Vasculitis Study Group cohort," Arthritis and Rheumatism, vol. 65, no. 1, pp. 270-281, 2013.

[5] Z. Cui, M.-H. Zhao, M. Segelmark, and T. Hellmark, "Natural autoantibodies to myeloperoxidase, proteinase 3, and the glomerular basement membrane are present in normal individuals," Kidney International, vol. 78, no. 6, pp. 590-597, 2010.

[6] A. Craven, J. Robson, C. Ponte et al., "ACR/EULAR-endorsed study to develop diagnostic and classification criteria for vasculitis (DCVAS)," Clinical and Experimental Nephrology, vol. 17, no. 5, pp. 619-621, 2013.

[7] K.-E. Sada, K. Amano, R. Uehara et al., "A nationwide survey on the epidemiology and clinical features of eosinophilic granulomatosis with polyangiitis (Churg-Strauss) in Japan," Modern Rheumatology, vol. 24, no. 4, pp. 640-644, 2014.

[8] L. Guillevin, C. Pagnoux, R. Seror, A. Mahr, L. Mouthon, and P. L. Toumelin, "The five-factor score revisited: assessment of prognoses of systemic necrotizing vasculitides based on the french vasculitis study group (FVSG) cohort," Medicine, vol. 90, no. 1, pp. 19-27, 2011.

[9] T. Miszalski-Jamka, W. Szczeklik, B. Sokołowska et al., "Noncorticosteroid immunosuppression limits myocardial damage and contractile dysfunction in eosinophilic granulomatosis with polyangiitis (churg-strauss syndrome)," Journal of the American College of Cardiology, vol. 65, no. 1, pp. 103-105, 2015.

[10] J. Seo, J. Song, D. Kim, D. Kang, and J. Song, "A case of loeffler's endocarditis associated with churg-strauss syndrome," Journal of Cardiovascular Ultrasound, vol. 18, no. 1, pp. 21-24, 2010.

[11] M. Cugno, A. V. Marzano, M. Lorini, V. Carbonelli, and A. Tedeschi, "Enhanced tissue factor expression by blood eosinophils from patients with hypereosinophilia: a possible link with thrombosis," PLoS ONE, vol. 9, no. 11, Article ID e111862, 2014.

[12] L. Mastalerz, M. Celinska-Lowenhoff, P. Krawiec, B. Batko, W. Tlustochowicz, and A. Undas, "Unfavorably altered fibrin clot properties in patients with eosinophilic granulomatosis with polyangiitis (churg-strauss syndrome): association with thrombin generation and eosinophilia," PLOS ONE, vol. 10, no. 11, Article ID e0142167, 2015.

[13] T. Lisitsyna, Z. Alekberova, P. Ovcharov, A. Volkov, J. Korsakova, and E. Nasonov, "Left ventricular intracardiac thrombus in a patient with Behçet disease successfully treated with immunosuppressive agents without anticoagulation: a case report and review of the literature," Rheumatology International, vol. 35, no. 11, pp. 1931-1935, 2015.

[14] S. Otto, H. R. Figulla, B. Goebel, P. Oelzner, and T. C. Poerner, "Churg-Strauss syndrome presenting with cardiac mass and right ventricular obstruction," Clinical Cardiology, vol. 32, no. 12, pp. E77-E79, 2009. 


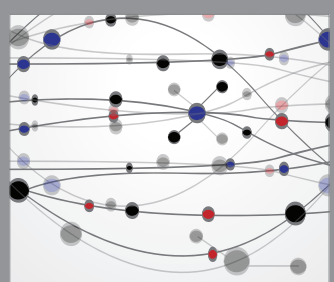

The Scientific World Journal
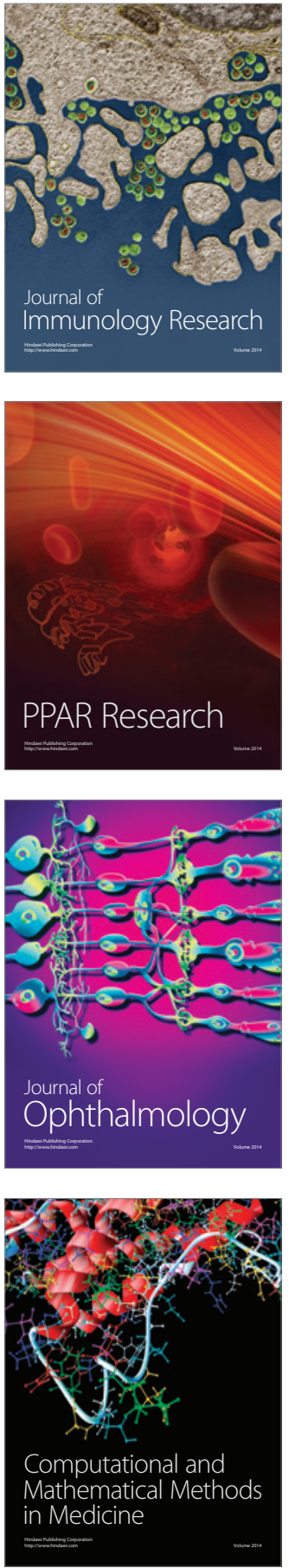

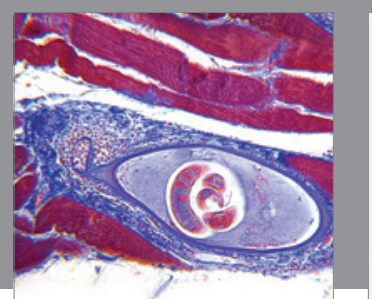

Gastroenterology Research and Practice
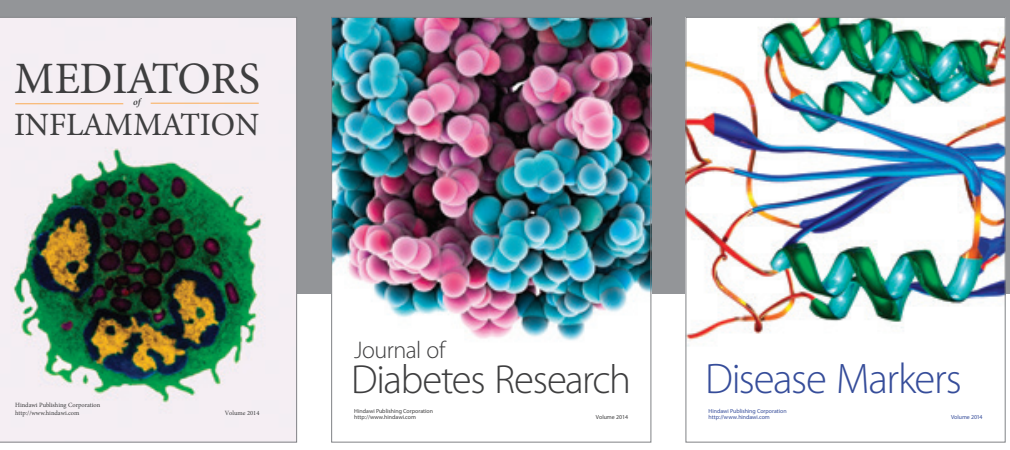

Disease Markers

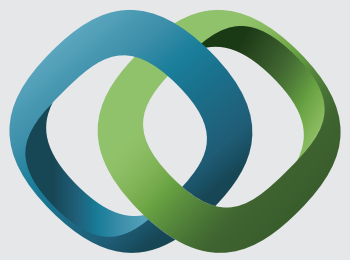

\section{Hindawi}

Submit your manuscripts at

https://www.hindawi.com
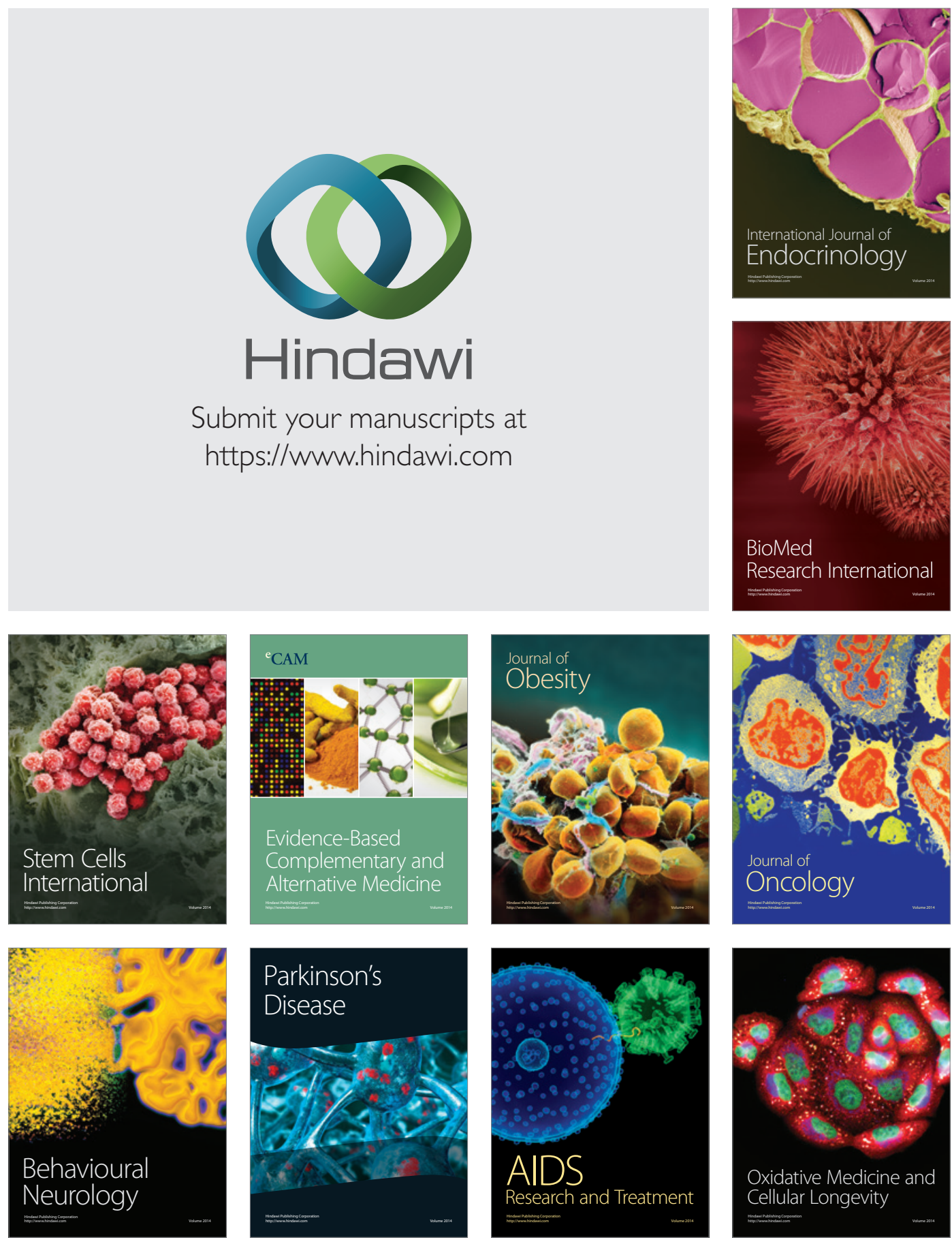